\title{
Observation of direction-dependent mechanical properties in the human brain with multi-excitation MR elastography
}

\author{
Aaron T. Anderson ${ }^{\mathrm{a}}$, Elijah E.W. Van Houten ${ }^{\mathrm{b}, \mathrm{c}}$, Matthew D.J. McGarry ${ }^{\mathrm{c}}$, \\ Keith D. Paulsen ${ }^{\mathrm{c}, \mathrm{d}}$, Joseph L. Holtrop ${ }^{\mathrm{e}, \mathrm{f}}$, Bradley P. Sutton ${ }^{\mathrm{e}, \mathrm{f}}$, John G. \\ Georgiadis $^{\mathrm{a}, \mathrm{g}}$, Curtis L. Johnson ${ }^{\mathrm{f}, \mathrm{h}}$ \\ ${ }^{a}$ Mechanical Science and Engineering Department, University of Illinois at \\ Urbana-Champaign, Urbana, Illinois 61801, USA \\ ${ }^{b}$ Département de génie mécanique, Université de Sherbrooke, Sherbrooke, Québec J1K2R1, \\ Canada \\ ${ }^{c}$ Thayer School of Engineering, Dartmouth College, Hanover, New Hampshire 03755, USA \\ ${ }^{d}$ Dartmouth-Hitchcock Medical Center, Lebanon, New Hampshire 03756, USA \\ ${ }^{e}$ Bioengineering Department, University of Illinois at Urbana-Champaign, Urbana, Illinois \\ 61801, USA \\ ${ }^{f}$ Beckman Institute for Advanced Science and Technology, University of Illinois at \\ Urbana-Champaign, Urbana, Illinois 61801, USA \\ ${ }^{g}$ Biomedical Engineering Department, Illinois Institute of Technology, Chicago, Illinois \\ 60616, USA \\ ${ }^{h}$ Department of Biomedical Engineering, University of Delaware, Newark, Delaware 19716, \\ $U S A$
}

\begin{abstract}
Magnetic resonance elastography (MRE) has shown promise in noninvasively capturing changes in mechanical properties of the human brain caused by neurodegenerative conditions. MRE involves vibrating the brain to generate shear waves, imaging those waves with MRI, and solving an inverse problem to determine mechanical properties. Despite the known anisotropic nature of brain tissue, the inverse problem in brain MRE is based on an isotropic mechanical model. In this study, distinct wave patterns are generated in the brain through the use of multiple excitation directions in order to characterize the potential impact of anisotropic tissue mechanics on isotropic inversion meth-
\end{abstract}

\footnotetext{
Email addresses: aandrsn3@illinois.edu (Aaron T. Anderson), eew.vanhouten@usherbrooke.ca (Elijah E.W. Van Houten), matthew.d.mcgarry@dartmouth.edu (Matthew D.J. McGarry), keith.d.paulsen@dartmouth.edu (Keith D. Paulsen), holtrop1@illinois.edu (Joseph L. Holtrop), bsutton@illinois.edu (Bradley P. Sutton), jgeorgia@iit.edu (John G. Georgiadis), clj@udel.edu (Curtis L. Johnson)
}

Preprint submitted to Elsevier

March 1, 2016

(C) 2016. This manuscript version is made available under the Elsevier user license http://www.elsevier.com/open-access/userlicense/1.0/ 
ods. Isotropic inversions of two unique displacement fields result in mechanical property maps that vary locally in areas of highly aligned white matter. Investigation of the corpus callosum, corona radiata, and superior longitudinal fasciculus, three highly-ordered white matter tracts, revealed differences in estimated properties between excitations of up to $33 \%$. Using diffusion tensor imaging to identify dominant fiber orientation of bundles, relationships between estimated isotropic properties and shear asymmetry are revealed. This study has implications for future isotropic and anisotropic MRE studies of white matter tracts in the human brain.

Keywords: magnetic resonance elastography; nonlinear inversion; anisotropic soft tissue; stiffness; human brain; white matter

\section{Introduction}

Neurodegeneration occurring in conditions such as Alzheimer's disease, amyotrophic lateral sclerosis, multiple sclerosis, and normal aging affects macroscale brain tissue characteristics due to microscale tissue changes. The alteration of the neuronal and glial matrix can result in detectable changes in physical material characteristics, including mechanical properties. Magnetic resonance elastography (MRE) is a technique for noninvasively assessing tissue viscoelasticity in vivo [1]. The mechanical contrast afforded by MRE has shown great promise in diagnosing and staging a variety of conditions, most notably chronic liver disease $[2,3]$, and MRE of the brain has recently revealed softening in a number of neurodegenerative conditions $[4,5,6,7,8,9]$.

Despite the success of brain MRE, most common methodological approaches adopt an oversimplified model of the complex tissue behavior that affects the accuracy and precision of the property measures. In particular, MRE typically assumes the material is isotropic, i.e. it does not exhibit direction-dependent properties, despite the fibrous structure of brain tissue, especially white matter (WM). A number of studies on animal brains suggest the necessity of considering anisotropic tissue properties in assessing the brain with MRE $[10,11,12,13,14$, 
15]. For instance, Velardi et al. [16] performed an ex vivo axial test of porcine WM and found that when stretched along the fiber direction stiffness appeared approximately three times greater than when stretched against the fibers. Feng et al. [10] performed shear tests on ex vivo lamb brains and found the shear modulus to be approximately $40 \%$ greater along the fiber than against. Similar behavior is expected in human brains given the shared fiber structure.

The adoption of an isotropic material model for brain MRE, in spite of the evidence supporting tissue anisotropy, is motivated by the need to maintain stability in solving the ill-posed inverse problem. Anisotropic models require additional material parameters to be estimated while enforcing isotropy reduces the burden on the inversion of noisy data. Though recent work has aimed to estimate anisotropic tissue properties with $\operatorname{MRE}[11,12,13,14,17,18,19$, 20], including specific WM tracts in the brain [7, 15, 21, 22, 23], the effect of WM anisotropy on traditional isotropic inversion schemes has yet to be fully characterized [24].

The present study aims to explore the differences in reconstructed isotropic mechanical properties of the brain in the presence of unique mechanical excitations. We aim to use a multi-excitation MRE setup to generate distinct shear patterns in the brain, and will evaluate our hypothesis that the directionality of shear strain relative to WM fiber orientation influences mechanical property estimation. Local fiber orientation will be determined using diffusion tensor imaging (DTI) in three major WM tracts of interest: corpus callosum (CC), corona radiata (CR), and superior longitudinal fasciculus (SLF).

\section{Methods}

A single 28-year-old healthy male subject participated in this study approved by the University of Illinois at Urbana-Champaign Institutional Review Board after providing written informed consent. The imaging protocol consisted of two MRE scans performed using two separate actuator setups, DTI, and a highresolution structural acquisition (MPRAGE). All scanning was performed on a 
Siemens 3 T Trio MRI scanner (Siemens Medical Solutions; Erlangen, Germany). Four repeats of the imaging protocol were performed on the same subject. These repeats were collected on different days, spread across four months.

\subsection{MRE Acquisition}

MRE displacement imaging used a 3D multislab, multishot spiral MRE sequence for generating 3D, full vector field complex displacement data at $50 \mathrm{~Hz}$ with $2 \times 2 \times 2 \mathrm{~mm}^{3}$ isotropic spatial resolution [25]. The specific imaging parameters for MRE include single shot in-plane spiral readout with SENSE $R=3$; ten slabs of eight $k_{z}$ sampling planes with $25 \%$ slab overlap; $T R / T E=1800 / 73 \mathrm{~ms}$; four (4) temporal phase offsets; and FOV $=240 \times 240 \times 120 \mathrm{~mm}$. Small strains $\left(\sim 10^{-5}\right)$ in the brain were induced through whole-head vibrations using a pneumatic actuator system (Resoundant, Inc., Rochester, MN). In order to create distinct excitation patterns, two passive drivers are positioned on the patient's head inside of the head coil: a soft pillow-like pad under the posterior of the head (Figure 1(a)) and a harder pad on the right side of the head (Figure 1(b)). The soft pad is often used for head MRE experiments [26, 27] due to its slim profile allowing it to fit comfortably in the standard MRI head coil. Additionally, the position posterior to the head results in good coupling to the skull through the weight of the head. The hard pad was used for the LR experiment to achieve adequate coupling with the head through contact with both the skull and the side of the head coil. The dimensions of the head coil precluded the use of soft pad for the LR excitation case. The MRE scan was performed twice with one driver activated for the entire imaging experiment at a time. The actuator driver is switched manually through a connection on the pneumatic tube between scans without altering the head position. As expected, the two excitation directions, referred to as anterior-posterior (AP) and left-right (LR), provide distinct deformation fields in the human brain (see Figure 1). In order to ensure optimal contact and sufficiently minimize involuntary movement of the head, foam pads were placed between the head and the MR coil on the subject's left side, and the driver adjacent to the right side of the head and the 
coil.

\subsection{Material Property Reconstruction}

Each of the individual displacement fields from MRE imaging were reconstructed into full brain material property maps using the finite element based non-linear inversion algorithm (NLI) $[28,29,30,31]$. NLI is based on an a priori model for mechanical tissue behavior, which in this case is a heterogeneous, isotropic, viscoelastic material model. This is governed by Navier's equation of the form

$$
\nabla \cdot\left(\mu\left(\nabla \tilde{\mathbf{u}}+\nabla \tilde{\mathbf{u}}^{T}\right)\right)+\nabla(\lambda \nabla \cdot \tilde{\mathbf{u}})=\rho \frac{\partial^{2} \tilde{\mathbf{u}}}{\partial t^{2}},
$$

where $\tilde{\mathbf{u}}$ is the $3 \mathrm{D}$ displacement field, $\lambda$ and $\mu$ are Lame's material constants, $\rho$ is the density (assumed to be $1000 \mathrm{~kg} / \mathrm{m}^{3}$ ), and $\nabla$ is the gradient operator. The complex displacement field is assumed to be at a steady-state of the form $\tilde{\mathbf{u}}(x, y, z, t)=\mathbf{u}(x, y, z) \exp (i \omega t)$ (where $\mathbf{u}(x, y, z)$ is complex), and the governing equation, Eq. (1), then simplifies to

$$
\nabla \cdot\left(\mu\left(\nabla \mathbf{u}+\nabla \mathbf{u}^{T}\right)\right)+\nabla(\lambda \nabla \cdot \mathbf{u})=-\rho \omega^{2} \mathbf{u}
$$

NLI employs a finite element representation of the object in order to compute an expected displacement field $\left(\mathbf{u}_{c}\right)$ from an estimate of the material properties $\left(\theta=\left[G^{\prime}, G^{\prime \prime}\right]\right)$. This is then compared to the measured displacement field $\left(\mathbf{u}_{m}\right)$, and the material property estimation is iteratively updated in order to minimize a mean-squared error cost function

$$
\Phi=\sum_{\Omega}\left\|\mathbf{u}_{c}(\theta)-\mathbf{u}_{m}\right\|^{2}
$$

where the summation is over the physical domain of interest, $\Omega$. The result of the reconstruction is a spatial map of the complex shear modulus $\mu=G=G^{\prime}+i G^{\prime \prime}$, where $G^{\prime}$ and $G^{\prime \prime}$ are the storage and loss modulus, respectively. To reduce the overall computational costs, minimization of Eq. (3) is reformatted in parallel as subzone subdomains of the full physical domain, $\Omega$. 


\subsection{Other Imaging}

DTI data was acquired in the same imaging session in a co-registered fashion with FOV and resolution matched to the MRE data. DTI parameters include: single-shot EPI with GRAPPA $R=2 ; T R / T E=8000 / 95 \mathrm{~ms} ; 30$ non-collinear encoding directions; $b=1000 \mathrm{~s} / \mathrm{mm}^{2}$. The diffusion tensor was processed using FMRIB Diffusion Toolkit (FDT) [32] to extract the voxel-wise diffusion eigenvectors. Finally, we acquired a $T_{1}$-weighted structural image using an MPRAGE sequence, which resulted in a $0.9 \times 0.9 \times 0.9 \mathrm{~mm}^{3}$ isotropic resolution $(T R / T I / T E=2000 / 900 / 2.2 \mathrm{~ms})$.

\subsection{Analysis}

\subsubsection{Computing Strain}

First the strain is calculated from MRE displacement fields to investigate the strain at each voxel to better understand how the strain state is related to the reconstructed properties from NLI between the two excitation directions. Isotropic tissue will behave the same under different shear modes; conversely, anisotropic material will respond differently in each independent property direction. The strain is computed from the MRE displacement at each temporal phase offset via

$$
\varepsilon_{i j}\left(x_{k}, t_{n}\right)=\frac{1}{2}\left(u_{i, j}+u_{j, i}\right)\left(x_{k}, t_{n}\right)
$$

where $x_{k}$ is the spatial coordinate and $t_{n}$ is the time at point $n$. The strain tensor is symmetric, and thus can be written as

$$
\begin{aligned}
\frac{1}{2}\left(u_{i, j}+u_{j, i}\right)\left(x_{k}, t_{n}\right)= & \\
= & {\left[\begin{array}{ccc}
u_{11} & \frac{1}{2}\left(u_{12}+u_{21}\right) & \frac{1}{2}\left(u_{13}+u_{31}\right) \\
\frac{1}{2}\left(u_{21}+u_{12}\right) & u_{22} & \frac{1}{2}\left(u_{23}+u_{32}\right) \\
\frac{1}{2}\left(u_{31}+u_{13}\right) & \frac{1}{2}\left(u_{32}+u_{23}\right) & u_{33}
\end{array}\right]\left(x_{k}, t_{n}\right)=} \\
& =\left[\begin{array}{ccc}
\varepsilon_{11} & \varepsilon_{12} & \varepsilon_{13} \\
\varepsilon_{12} & \varepsilon_{22} & \varepsilon_{23} \\
\varepsilon_{13} & \varepsilon_{23} & \varepsilon_{33}
\end{array}\right]\left(x_{k}, t_{n}\right),
\end{aligned}
$$


The root-mean-square (RMS),

$$
\varepsilon_{r m s, i j}\left(x_{k}\right)=\sqrt{\frac{1}{N_{t}} \sum_{n=1}^{N_{t}}\left[\varepsilon_{i j}\left(x_{k}, t_{n}\right)\right]^{2}},
$$

is computed to obtain a magnitude of the oscillatory shear and normal strains across the temporal phase offsets. Maps of the RMS values for the shear components of the strain tensor are shown in Figure 2. It is important to note the strain calculations were performed independent from NLI to reduce bias from the specific NLI formulation.

\subsubsection{Fiber-Tract Reference Frame}

NLI is formulated with respect to the imaging reference frame, and all inversions in this work are completed in this reference frame. However, we seek to understand how isotropic MRE reconstructions of an expected anisotropic material, and thus we want to compute the strain state relative to the direction of neuronal fiber bundles, which represent the presumed axis of symmetry in a transversely isotropic material $[12,15]$. The strain tensor at each voxel, Eq. (4), can be transformed from the lab (imaging) reference frame of the MRE images, $\hat{x}^{l}$, into the axonal fiber-tract reference frame, $\hat{x}^{f}$, by constructing a transformation matrix from the DTI eigenvectors $\left(V_{j}\right)$. The DTI eigenvectors, assumed to be the fiber orientation reference frame in typical DTI, are the local coordinate system of the voxel, where $\hat{x}_{j}^{f}=V_{j}$ and $\hat{x}_{1}^{f}$ corresponds to the principal fiber

direction, while $\hat{x}_{2}^{f}$ and $\hat{x}_{3}^{f}$ correspond to the two directions perpendicular to the fiber-tracts. The transformation tensor is constructed via the cosine of the angle between the lab and fiber coordinates:

$$
[T]=\cos \left(\hat{x}_{i}^{l}, \hat{x}_{j}^{f}\right)
$$

The transformation of the strains in the lab coordinates into the fiber-tract's local orientation is then computed for each time point

$$
\left[\varepsilon^{f}\right]=\left[T^{T}\right]\left[\varepsilon^{l}\right][T]
$$


and the RMS of strain is calculated in the same fashion as in section 2.4.1. In order to highlight the asymmetry of shear strains relative to fiber-tracts and thus the likely anisotropic response, the shear strains in planes parallel, $\varepsilon_{12}^{f}$ and $\varepsilon_{13}^{f}$, and perpendicular, $\varepsilon_{23}^{f}$, to the dominant fiber direction, $\hat{x}_{1}^{f}$, are compared via

$$
\varepsilon_{\| / \perp}=\frac{\frac{1}{2}\left(\varepsilon_{12}^{f}+\varepsilon_{13}^{f}\right)}{\varepsilon_{23}^{f}} .
$$

The spatial, multiple experiment averages of Eq. (9) are computed for various regions of the brain in Table 3 , with ()* indicating statistically significant differences determined by a paired $t$-test.

\subsubsection{Registration}

The images from a given scanning session are acquired in a co-registered fashion (both MRE scans and DTI scan). The DTI acquired during each experiment is used to register these data for identifying the WM regions of interest through an atlas [33] using the FSL TBSS tool [34]. The WM tracts investigated in this study are the corpus callosum (CC), corona radiata $(\mathrm{CR})$, and superior longitudinal fasciculus (SLF). Additionally, the FAST tool in FSL [35] segmented the MPRAGE to delineate the global WM region from the images for comparison with the individual tract (CC, CR, and SLF) statistics.

\section{Results}

An example of the differences in displacement fields for a subject undergoing two different excitations is shown in Figure 1. The displacement fields show a different displacement pattern for each excitation, particularly for the outof-plane direction. AP excitation results in patterns with left-right symmetry, while the LR excitation exhibits anterior-posterior symmetry, as expected. The uniqueness of patterns is indicative of differentially excited shear modes, which results in distinct strain fields, and the distinct fields are necessary for investigating direction-dependent reponses. Displacement fields from both AP (soft pad) and LR (hard pad) excitation were of sufficiently high quality for inversion, 
with octahedral shear strain-based SNR (OSS-SNR) for all displacement fields greater than $3[36]$.

Figure 2 shows the RMS strain of the displacement field for the same axial slice as in Figure 1. At the posterior of the subject's head, both excitation directions have the strongest strain magnitudes due to the shear source at the skull and falx. However, the strain throughout the brain has voxel-wise spatial variation differing with excitation direction, especially as the shear propagates towards the center. The AP excitation shows the outline of the ventricle region in each of the strain components, which is less visible in LR excitation. The lack of definition in strain patterns for LR at the center may be due to the excitation wave passing across the long side of the ventricles.

Figure 3 presents an example of the reconstructed material property maps for the storage $\left(G^{\prime}\right)$ and loss $\left(G^{\prime \prime}\right)$ moduli at an axial slice encompassing regions adjacent to the lateral ventricles, the $\mathrm{CC}$, and the $\mathrm{CR}$. Note that the change of excitation orientation results in voxel-wise differences in $G^{\prime}$ and $G^{\prime \prime}$ of up to approximately $2 \mathrm{kPa}$. Such large differences between the AP and LR excitation appear mostly in areas with high DTI fractional anisotropy (FA): $\overline{F A}_{C C}=0.70$, $\overline{F A}_{C R}=0.51$, and $\overline{F A}_{S L F}=0.50$.

A summary of the mean and standard deviation of reconstructed $G^{\prime}$ and $G^{\prime \prime}$ moduli across the repeated experiments for the entire brain, global WM, CC, CR, and SLF are given in Tables 1 and 2. Values are reported for each excitation and the relation between the two, with ()* indicating statistically significant differences determined by a paired $t$-test. The whole brain averages for $G^{\prime}$ and $G^{\prime \prime}$ for both excitation directions are consistent with published values and exhibit very low standard deviations across repeated experiments $(\leq 2 \%)$, indicating excellent reproducibility, in agreement with other brain MRE studies using NLI $[26,37]$. Similarly, the global WM region exhibits consistent $G^{\prime}$ and $G^{\prime \prime}$ between excitations and comparable reproducibility.

Additionally, both global regions exhibit comparable and reproducible ratios of shear parallel to perpendicular with respect to dominant fiber-tracts direction, 
$\varepsilon_{\| / \perp}^{f}$ (Eq. (9)), as summarized in Table 3. This is expected as the global regions do not isolate specific, ordered fiber-tracts.

The spatial mean values for $G^{\prime}$ and $G^{\prime \prime}$ in each WM tract for each experiment are plotted in Figure 4 against the spatial mean values for $\varepsilon_{\| / \perp}^{f}$. The CC shows the largest difference between reconstructed $G^{\prime}$ and $G^{\prime \prime}, 23 \%$ and $26 \%$ on average, for the two excitation experiments. Both $G^{\prime}$ and $G^{\prime \prime}$ are greater in LR excitation experiments, which corresponds to a greater $\varepsilon_{\| / \perp}^{f}$. The CR similarly exhibits greater $G^{\prime}$ and $G^{\prime \prime}$ in LR excitation; although, in contrast, there is a slight decrease in $\varepsilon_{\| / \perp}^{f}$. The SLF shows no discernible difference in either $G^{\prime}$ or $G^{\prime \prime}$, potentially due to the variability of $\varepsilon_{\| / \perp}^{f}$ in both LR and AP excitations.

\section{Discussion}

The AP and LR excitations each provide distinct deformation states in the human brain, as evidenced by both the displacement fields (Figure 1) and the computed RMS shear strain fields (Figure 2). The unique strain fields are a necessary start for characterizing how MRE interprets shear strain applied to anisotropic media. The discrepancies (locally over $2 \mathrm{kPa}$ ) in reconstructed $G^{\prime}$ and $G^{\prime \prime}$ (Figure 3) show the inherent limitation of the isotropic assumption used for the NLI material model when investigating anisotropic media.

The mean and standard deviation statistics (Tables 1, 2, and 3) for results in the whole brain, global WM, and individual WM regions (CC, CR, and SLF tracts) help provide initial context for the local effect of shear strain mode on the reconstructed material properties. The differences between excitation for global $G^{\prime}, G^{\prime \prime}$, and shear strain $\left(\varepsilon_{\| / \perp}^{f}\right)$ are $<1.5 \%$ across all repeats. The findings for global WM are similar, with non-significant differences of $<3.5 \%$ between excitations.

Significant differences in reconstructed material properties occur in areas of the highest local anisotropy, as well as the greatest difference in shear strain ratio. The $\varepsilon_{\| / \perp}^{f}$ in the CC differed between the two excitations by $22.3 \%$ (normalized) and both the reconstructed $G^{\prime}$ and $G^{\prime \prime}$ had over $20 \%$ normalized dif- 
ference, $23 \%$ and $26 \%$, respectively. The stark difference in properties in the $\mathrm{CC}$ is not surprising, as the CC has a highly-aligned, well-ordered fiber structure [38] known to exhibit mechanical anisotropy [16, 39], and the two excitation fields provide sufficiently different strain states expected to illuminate directiondependent properties in MRE. This is highlighted by Figure 4(a), where the CC properties show a linear increase in both $G^{\prime}$ and $G^{\prime \prime}$ with increasing $\varepsilon_{\| / \perp}^{f}$. In other words, the tissue appears stiffer when exposed to strains in planes parallel to the dominant fiber axis. This apparent relationship suggests that as the disparity in axonal shear components changes, the isotropic MRE inversion effectively "sees" a different underlying material.

The relationship between excitation mode and reconstructed MRE properties is less clear in the CR. In this case, we found a much smaller difference in shear strain ratio between excitations (8.8\%). This translated in to a small, non-significant difference in $G^{\prime}(11 \%)$, but a large, significant difference in $G^{\prime \prime}$ (33\%). Most interestingly, the apparent relationship between $G^{\prime}$ and $G^{\prime \prime}$ in the CR with $\varepsilon_{\| / \perp}^{f}$ (Figure 4(b)) is opposite from that of the CC in that $G^{\prime}$ and $G^{\prime \prime}$ decrease with increasing $\varepsilon_{\| / \perp}^{f}$. How the magnitude and the relationship between MRE properties and strain state in the CR differs from those in the CC may be explained by the more complex fiber structure of the CR. The CR encompasses fibers that fan in the superior-inferior direction as they approach the cortex, and also include populations of crossing fibers. The disparity in fiber structure has been previously used to understand MRE properties in the CC and CR [21], and may be underpinning the relationships reported here.

We did not observe differences in $G^{\prime}$ and $G^{\prime \prime}$ for the SLF, even though the SLF is also a large, well-ordered WM tract. We believe that the lack of significant differences in MRE properties between excitation is due to the strain fields themselves not being significantly different. The values for $\varepsilon_{\| / \perp}^{f}$ in the SLF exhibited higher standard deviations across repeats, even though the AP excitation had shown high reproducibility in other measures. This, in turn, resulted in poorer reproducibility of $G^{\prime}$ and $G^{\prime \prime}$ measures in the SLF that likely contributed to the lack of detectable significant difference. This suggests the 
strain fields towards the periphery of the brain generated by both excitation directions are very sensitive to the excitation mode, but that they are associated with consistent shear modes in the deeper WM regions.

The scenario of applying unique shear modes to characterize anisotropic material is similar to applying any type of mechanical tests with forcing in different directions, and has previously been used in MRE experiments on phantoms to characterize anisotropic effects $[40,41,42,43]$. In these works, the shear direction is carefully controlled and applied either along or against the known directions of material symmetry. In the brain, such control over shear direction is not feasible due to poor mechanical transmission through the skull, and also because the material anisotropy is itself spatially-varying. In this work, we use two distinct excitation directions and achieved success in generating shear modes sensitive to material anisotropy in two large WM tracts, the CC and the $\mathrm{CR}$, and not in another, the SLF. Future works could incorporate more excitation modes to better capture the behavior of all WM regions in the brain and studies to identify commonalities in WM behavior across a population.

Anisotropic MRE inversion schemes may benefit from adopting a multiexcitation approach. Previous anisotropic methods have aimed to reconstruct direction-dependent mechanical properties from a single deformation field through the incorporation of prior knowledge of material symmetry [11, 12, 14, 15, 17]. While the results from these methods appear promising [13, 22, 44], they may suffer in characterizing the heterogeneous anisotropy of the human brain if the excited shear mode is not judiciously chosen to account for material symmetries and sensitive to the material complex properties. By incorporating multiple excitation fields with sensitivities, anisotropic inversions may be stabilized during the reconstruction of multiple material constants. For example, Tweten et al. [42] and Schmidt et al. [43] performed controlled actuation studies on numerical phantoms[42], physical phantoms, and ex vivo tissue[43]. Both studies are important to anisotropic MRE because they show how a three parameter incompressible transversely isotropic material model can capture more information than the typical transversely isotropic model, including fiber stretch. The 
work in this study, in contrast, did not take into account the polarization of shear waves, but, as we move to higher order models, we should be careful to consider the potential effects of fiber stretching.

Finally, it is important to consider the implications of our findings on the interpretation of MRE results in WM tracts from traditional, single-frequency experiments. While we report that excitation mode and the amount of shear strain parallel and perpendicular to the plane containing the dominant fiber direction can result in significant differences in estimated mechanical properties, we, also, note that results from the AP excitation alone are very repeatable. AP excitation is the most common type of excitation used in brain MRE, generally achieved through pneumatic actuation of a soft pillow $[4,45]$ or a head rocker driven by remote electromechanical actuation $[25,46,47]$. LR excitation from a bite bar $[48,49,50]$, lateral pneumatic actuation [51], or scanner table vibration [52] is less commonly employed. In this case, we can expect that the use of AP excitation will result in highly repeatable measurements of WM tracts, as has been reported previously [21, 47], and that these measurements should be considered less sensitive to the excitation mode.

\section{Conclusions}

Material testing of animal brains has shown the mechanical anisotropy of brain tissue is significant and the human brain is expected to exhibit similar anisotropic properties. However, MRE methods have typically adopted an isotropic material model for considering the brain, thus ignoring directiondependent material properties expected to be present in WM. In this study, we performed multi-excitation MRE experiments and observed distinct differences in reconstructed isotropic material properties of well-ordered WM tracts due to excitation of unique shear modes. The differences arise from the anisotropic medium being sheared in different directions and the isotropic reconstruction "seeing" different properties depending on whether the material is sheared in planes parallel or perpendicular to the fibers. Through the use of both AP and 
LR excitations, we observed differences in reconstructed MRE properties, $G^{\prime}$ and $G^{\prime \prime}$, of up to $33 \%$ in WM tracts, while finding no difference in global properties. These findings have implications for future experiments and methodological developments targeting the reconstruction of anisotropic mechanical properties of WM, and may provide additional contrast for elucidating microstructural processes of neurodegeneration.

\section{Acknowledgments}

Support for ATA and JGG was provided by NSF grant CMMI-1437113. Partial support was provided by the Biomedical Imaging Center of the Beckman Institute for Advanced Science and Technology at the University of Illinois at Urbana-Champaign (UIUC), NIH grant R01-EB018230, and NIH/NIBIB grant R01-EB001981. This research is part of the Blue Waters sustained-petascale computing project, which is supported by the National Science Foundation (awards OCI-0725070 and ACI-1238993) and the state of Illinois. Blue Waters is a joint effort of the UIUC and its National Center for Supercomputing Applications. Additionally, computations were made on the supercomputer Mammouth parallèle 2 from the Université de Sherbrooke, managed by Calcul Québec and Compute Canada. The operation of this supercomputer is funded by the Canada Foundation for Innovation (CFI), NanoQuébec, RMGA and the Fonds de recherche du Québec - Nature et technologies (FRQ-NT).

\section{References}

[1] R. Muthupillai, D. J. Lomas, P. J. Rossman, J. F. Greenleaf, A. Manduca, R. L. Ehman, Magnetic resonance elastography by direct visualization of 
propagating acoustic strain waves, Science (New York, N.Y.) 269 (5232) (1995) 1854-1857.

[2] S. K. Venkatesh, M. Yin, R. L. Ehman, Magnetic resonance elastography of liver: Technique, analysis, and clinical applications, Journal of Magnetic Resonance Imaging 37 (3) (2013) 544-555.

[3] M. Yin, K. J. Glaser, J. A. Talwalkar, J. Chen, A. Manduca, R. L. Ehman, Hepatic MR Elastography: Clinical Performance in a Series of 1377 Consecutive Examinations., Radiology (2015) 142141.

[4] M. C. Murphy, J. R. Huston, C. R. J. Jack, K. J. Glaser, A. Manduca, J. P. Felmlee, R. L. Ehman, Decreased brain stiffness in Alzheimer's disease determined by magnetic resonance elastography., Journal of Magnetic Resonance Imaging 34 (3) (2011) 494-498.

[5] K.-J. Streitberger, I. Sack, D. Krefting, C. Pfüller, J. Braun, F. Paul, J. Wuerfel, Brain Viscoelasticity Alteration in Chronic-Progressive Multiple Sclerosis, PLoS ONE 7 (1) (2012) e29888.

[6] K.-J. Streitberger, E. Wiener, J. Hoffmann, F. B. Freimann, D. Klatt, J. Braun, K. Lin, J. McLaughlin, C. Sprung, R. Klingebiel, I. Sack, In vivo viscoelastic properties of the brain in normal pressure hydrocephalus, NMR in Biomedicine 24 (4) (2011) 385-392.

[7] A. Arani, M. C. Murphy, K. J. Glaser, A. Manduca, D. S. Lake, S. A. Kruse, C. R. Jack, R. L. Ehman, J. Huston, Measuring the effects of aging and sex on regional brain stiffness with MR elastography in healthy older adults, NeuroImage 111 (2015) 59-64.

[8] A. Lipp, R. Trbojevic, F. Paul, A. Fehlner, S. Hirsch, M. Scheel, C. Noack, J. Braun, I. Sack, Cerebral magnetic resonance elastography in supranuclear palsy and idiopathic Parkinson's disease, NeuroImage : Clinical 3 (2013) 381-387. 
[9] J. R. Huston, M. C. Murphy, B. F. Boeve, N. Fattahi, A. Arani, K. J. Glaser, A. Manduca, D. T. Jones, R. L. Ehman, Magnetic resonance elastography of frontotemporal dementia., Journal of Magnetic Resonance Imaging.

[10] Y. Feng, R. J. Okamoto, R. Namani, G. M. Genin, P. V. Bayly, Measurements of mechanical anisotropy in brain tissue and implications for transversely isotropic material models of white matter, Journal of the Mechanical Behavior of Biomedical Materials 23 (C) (2013) 117-132.

[11] R. Sinkus, M. Tanter, S. Catheline, J. Lorenzen, C. Kuhl, E. Sondermann, M. Fink, Imaging anisotropic and viscous properties of breast tissue by magnetic resonance-elastography, Magnetic Resonance in Medicine 53 (2) (2005) 372-387.

[12] E. C. Qin, R. Sinkus, G. Geng, S. Cheng, M. Green, C. D. Rae, L. E. Bilston, Combining MR elastography and diffusion tensor imaging for the assessment of anisotropic mechanical properties: A phantom study, Journal of Magnetic Resonance Imaging 37 (1) (2012) 217-226.

[13] E. C. Qin, L. Jugé, S. A. Lambert, V. Paradis, R. Sinkus, L. E. Bilston, In Vivo Anisotropic Mechanical Properties of Dystrophic Skeletal Muscles Measured by Anisotropic MR Elastographic Imaging: The mdx Mouse Model of Muscular Dystrophy, Radiology 273 (3) (2014) 726-735.

[14] A. J. Romano, P. B. Abraham, P. J. Rossman, J. A. Bucaro, R. L. Ehman, Determination and analysis of guided wave propagation using magnetic resonance elastography, Magnetic Resonance in Medicine 54 (4) (2005) 893-900.

[15] A. Romano, M. Scheel, S. Hirsch, J. Braun, I. Sack, In vivo waveguide elastography of white matter tracts in the human brain, Magnetic Resonance in Medicine 68 (5) (2012) 1410-1422. 
[16] F. Velardi, F. Fraternali, M. Angelillo, Anisotropic constitutive equations and experimental tensile behavior of brain tissue, Biomechanics and Modeling in Mechanobiology 5 (1) (2005) 53-61.

[17] J. Guo, S. Hirsch, M. Scheel, J. Braun, I. Sack, Three-parameter shear wave inversion in MR elastography of incompressible transverse isotropic media: Application to in vivo lower leg muscles, Magnetic Resonance in Medicine (2015) 1-9.

[18] S. Ipek-Ugay, T. Driessle, M. Ledwig, J. Guo, S. Hirsch, I. Sack, J. Braun, Tabletop magnetic resonance elastography for the measurement of viscoelastic parameters of small tissue samples, Journal of Magnetic Resonance 251 (2015) 13-18.

[19] J. A. Smith, A. J. Lawrence, J. F. Greenleaf, R. L. Ehman, S. A. Kruse, M. A. Dresner, A. Manduca, Tissue characterization using magnetic resonance elastography: preliminary results*, Physics in Medicine and Biology 45 (6) (2000) 1579-1590.

[20] J. B. Weaver, T. B. Miller, P. R. Perrinez, M. M. Doyley, H. Wang, Y. Y. Cheung, J. S. Wrobel, R. J. Comi, F. E. Kennedy, K. D. Paulsen, MR elastographic methods for the evaluation of plantar fat pads: preliminary comparison of the shear modulus for shearing deformation and compressive deformation in normal subjects, in: A. Manduca, A. A. Amini (Eds.), Medical Imaging 2006: Physiology, Function, and Structure from Medical Images, 2006, pp. 1-10.

[21] C. L. Johnson, M. D. J. McGarry, A. A. Gharibans, J. B. Weaver, K. D. Paulsen, H. Wang, W. C. Olivero, B. P. Sutton, J. G. Georgiadis, Local mechanical properties of white matter structures in the human brain, NeuroImage 79 (2013) 145-152.

[22] A. Romano, J. Guo, T. Prokscha, T. Meyer, S. Hirsch, J. Braun, I. Sack, M. Scheel, In vivo waveguide elastography: Effects of neurodegenera- 
tion in patients with amyotrophic lateral sclerosis, Magnetic Resonance in Medicine 72 (6) (2014) 1755-1761.

[23] G. Geng, M. Green, C. Rae, R. Sinkus, R. G. Henry, L. E. Bilston, Diffusion Tensor Imaging Enhanced Anisotropic MRE of the Brain, in: 21st Annual Meeting of ISMRM, 2013.

[24] A. T. Anderson, C. L. Johnson, J. L. Holtrop, M. D. J. McGarry, E. E. W. Van Houten, K. D. Paulsen, B. P. Sutton, J. G. Georgiadis, Property Differences in White Matter Structures due to Distinct Wave Propagation Directions in MR Elastography, in: 21st Annual Meeting of ISMRM, 2015.

[25] C. L. Johnson, J. L. Holtrop, M. D. J. McGarry, J. B. Weaver, K. D. Paulsen, J. G. Georgiadis, B. P. Sutton, 3D multislab, multishot acquisition for fast, whole-brain MR elastography with high signal-to-noise efficiency, Magnetic Resonance in Medicine 71 (2) (2013) 477-485.

[26] M. C. Murphy, J. Huston, C. R. Jack, K. J. Glaser, M. L. Senjem, J. Chen, A. Manduca, J. P. Felmlee, R. L. Ehman, Measuring the Characteristic Topography of Brain Stiffness with Magnetic Resonance Elastography, PLoS ONE 8 (12) (2013) e81668.

[27] H. Schwarb, C. L. Johnson, M. D. J. McGarry, N. J. Cohen, Medial temporal lobe viscoelasticity and relational memory performance, NeuroImage (2016) $1-27$.

[28] E. E. W. Van Houten, K. D. Paulsen, M. I. Miga, F. E. Kennedy, J. B. Weaver, An overlapping subzone technique for MR-based elastic property reconstruction, Magnetic Resonance in Medicine 42 (4) (1999) 779-786.

[29] E. E. W. Van Houten, M. I. Miga, J. B. Weaver, F. E. Kennedy, K. D. Paulsen, Three-dimensional subzone-based reconstruction algorithm for MR elastography, Magnetic Resonance in Medicine 45 (5) (2001) 827-837.

[30] E. E. W. Van Houten, D. v. Viviers, M. D. J. McGarry, P. R. Perriñez, I. I. Perreard, J. B. Weaver, K. D. Paulsen, Subzone based magnetic resonance 
elastography using a Rayleigh damped material model, Medical Physics 38 (4) (2011) 1993-2004.

[31] M. D. J. McGarry, E. E. W. Van Houten, C. L. Johnson, J. G. Georgiadis, B. P. Sutton, J. B. Weaver, K. D. Paulsen, Multiresolution MR elastography using nonlinear inversion, Medical Physics 39 (10) (2012) 6388-6396.

[32] T. E. J. Behrens, M. W. Woolrich, M. Jenkinson, H. Johansen-Berg, R. G. Nunes, S. Clare, P. M. Matthews, J. M. Brady, S. M. Smith, Characterization and propagation of uncertainty in diffusion-weighted MR imaging., Magnetic Resonance in Medicine 50 (5) (2003) 1077-1088.

[33] S. Mori, K. Oishi, H. Jiang, L. Jiang, X. Li, K. Akhter, K. Hua, A. V. Faria, A. Mahmood, R. Woods, A. W. Toga, G. B. Pike, P. R. Neto, A. Evans, J. Zhang, H. Huang, M. I. Miller, P. van Zijl, J. Mazziotta, Stereotaxic white matter atlas based on diffusion tensor imaging in an ICBM template., NeuroImage 40 (2) (2008) 570-582.

[34] S. M. Smith, M. Jenkinson, H. Johansen-Berg, D. Rueckert, T. E. Nichols, C. E. Mackay, K. E. Watkins, O. Ciccarelli, M. Z. Cader, P. M. Matthews, T. E. J. Behrens, Tract-based spatial statistics: Voxelwise analysis of multisubject diffusion data, NeuroImage 31 (4) (2006) 1487-1505.

[35] Y. Zhang, M. Brady, S. Smith, Segmentation of brain MR images through a hidden Markov random field model and the expectation-maximization algorithm, IEEE Transactions on Medical Imaging 20 (1) (2001) 45-57.

[36] M. D. J. McGarry, E. E. W. Van Houten, P. R. Perriñez, A. J. Pattison, J. B. Weaver, K. D. Paulsen, An octahedral shear strain-based measure of SNR for 3D MR elastography, Physics in Medicine and Biology 56 (13) (2011) N153-N164.

[37] C. L. Johnson, H. Schwarb, M. D. J. McGarry, B. P. Sutton, N. J. Cohen, Viscoelasticity of Subcortical Gray Matter Structures, in: 21st Annual Meeting of ISMRM, 2015. 
[38] F. Aboitiz, J. Montiel, One hundred million years of interhemispheric communication: the history of the corpus callosum., Braz J Med Biol Res 36 (4) (2003) 409-420.

[39] E. Mace, I. Cohen, G. Montaldo, R. Miles, M. Fink, M. Tanter, In vivo mapping of brain elasticity in small animals using shear wave imaging, IEEE Transactions on Medical Imaging 30 (3) (2011) 550-558.

[40] R. Namani, M. D. Wood, S. E. Sakiyama-Elbert, P. V. Bayly, Anisotropic mechanical properties of magnetically aligned fibrin gels measured by magnetic resonance elastography., Journal of Biomechanics 42 (13) (2009) 20472053.

[41] I. M. Perreard, A. J. Pattison, M. Doyley, M. D. J. McGarry, Z. Barani, E. E. Van Houten, J. B. Weaver, K. D. Paulsen, Effects of frequencyand direction-dependent elastic materials on linearly elastic MRE image reconstructions., Physics in Medicine and Biology 55 (22) (2010) 68016815.

[42] D. J. Tweten, R. J. Okamoto, J. L. Schmidt, J. R. Garbow, P. V. Bayly, Estimation of material parameters from slow and fast shear waves in an incompressible, transversely isotropic material, Journal of Biomechanics 48 (15) (2015) 4002-4009.

[43] J. L. Schmidt, D. J. Tweten, A. N. Benegal, C. H. Walker, T. E. Portnoi, R. J. Okamoto, J. R. Garbow, P. V. Bayly, Magnetic resonance elastography of slow and fast shear waves illuminates differences in shear and tensile moduli in anisotropic tissue, Journal of Biomechanics (2016) 1-8.

[44] M. A. Green, G. Geng, E. Qin, R. Sinkus, S. C. Gandevia, L. E. Bilston, Measuring anisotropic muscle stiffness properties using elastography., NMR in Biomedicine 26 (11) (2013) 1387-1394.

[45] D. Klatt, C. L. Johnson, R. L. Magin, Simultaneous, multidirectional acquisition of displacement fields in magnetic resonance elastography of the 
in vivo human brain, Journal of Magnetic Resonance Imaging 42 (2) (2015) 297-304.

[46] I. Sack, B. Beierbach, U. Hamhaber, D. Klatt, J. Braun, Non-invasive measurement of brain viscoelasticity using magnetic resonance elastography, NMR in Biomedicine 21 (3) (2008) 265-271.

[47] J. Guo, S. Hirsch, A. Fehlner, S. Papazoglou, M. Scheel, J. Braun, I. Sack, Towards an Elastographic Atlas of Brain Anatomy, PLoS ONE 8 (8) (2013) e71807.

[48] S. A. Kruse, G. H. Rose, K. J. Glaser, A. Manduca, J. P. Felmlee, C. R. Jack, R. L. Ehman, Magnetic resonance elastography of the brain, NeuroImage 39 (1) (2008) 231-237.

[49] M. A. Green, L. E. Bilston, R. Sinkus, In vivo brain viscoelastic properties measured by magnetic resonance elastography, NMR in Biomedicine 21 (7) (2008) 755-764.

[50] U. Hamhaber, D. Klatt, S. Papazoglou, M. Hollmann, J. Stadler, I. Sack, J. Bernarding, J. Braun, In vivo magnetic resonance elastography of human brain at $7 \mathrm{~T}$ and $1.5 \mathrm{~T}$, Journal of Magnetic Resonance Imaging 32 (3) (2010) $577-583$.

[51] P. Latta, M. L. H. Gruwel, P. Debergue, B. Matwiy, U. N. SbotoFrankenstein, B. Tomanek, Convertible pneumatic actuator for magnetic resonance elastography of the brain, Magnetic Resonance Imaging 29 (1) (2011) 147-152.

[52] D. Gallichan, M. D. Robson, A. Bartsch, K. L. Miller, TREMR: Tableresonance elastography with MR, Magnetic Resonance in Medicine 62 (3) (2009) 815-821. 


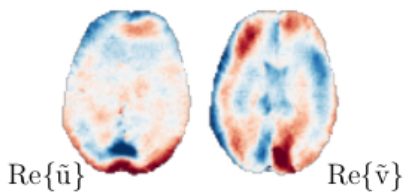

(a) AP

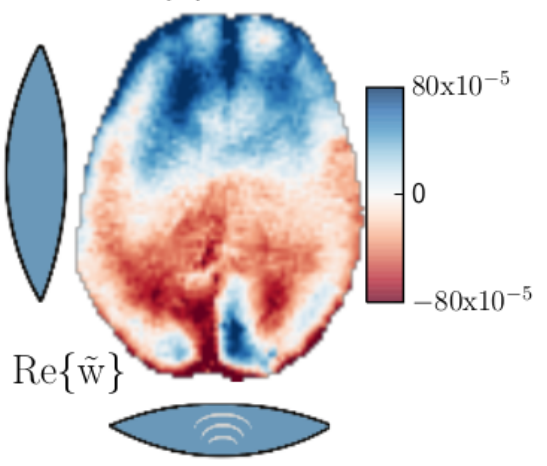

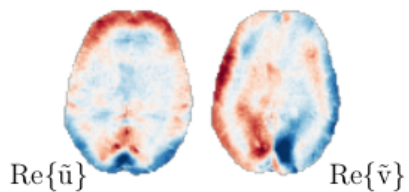

(b) LR

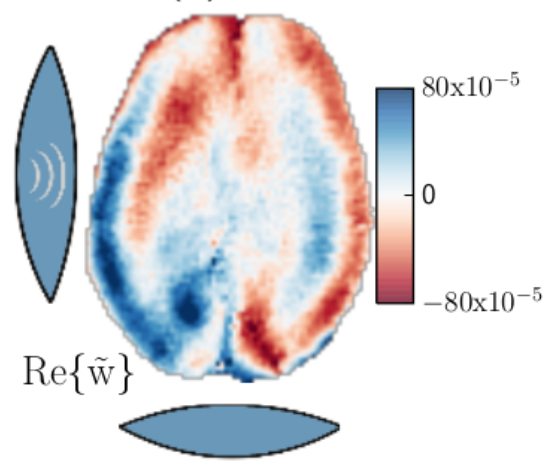

Figure 1: The real component of the complex displacement fields $(\tilde{u}=\{\tilde{u}, \tilde{v}, \tilde{w}\})$ generated by excitation from two different actuator setups: (a) anterior-posterior (AP) and (b) left-right (LR). The two excitations produce distinctly different patterns, particularly for the out-ofplane direction. AP excitation results in patterns with left-right symmetry, while the LR excitation exhibits anterior-posterior symmetry, as expected.

Table 1: Summary of mean and standard deviation for the reconstructed storage modulus, $G^{\prime}$, across experimental repeats of spatial averages of the whole brain, global WM, and selected WM regions (CC, CR, and SLF); ()* represents $p<0.05$ for the normalized differences; $\overline{(,)}$ is a two value average.

\begin{tabular}{cccccc} 
& Whole Brain & WM & CC & CR & SLF \\
\hline $\bar{G}_{A P}^{\prime}$ & $2.45(1.1 \%)$ & $2.68(1.2 \%)$ & $2.24(1.3 \%)$ & $3.03(1.6 \%)$ & $2.85(1.9 \%)$ \\
\hline $\bar{G}_{L R}^{\prime}$ & $2.48(1.6 \%)$ & $2.74(1.7 \%)$ & $2.81(4.2 \%)$ & $3.32(5.7 \%)$ & $2.82(5.2 \%)$ \\
\hline$\frac{\left(\bar{G}_{A P}^{\prime}-\bar{G}_{L R}^{\prime}\right)}{\left(G_{A P}^{\prime}, G_{L R}^{\prime}\right)}$ & $-1.2 \%$ & $-2.2 \%$ & $-23 \% *$ & $-11 \%$ & $1.3 \%$
\end{tabular}




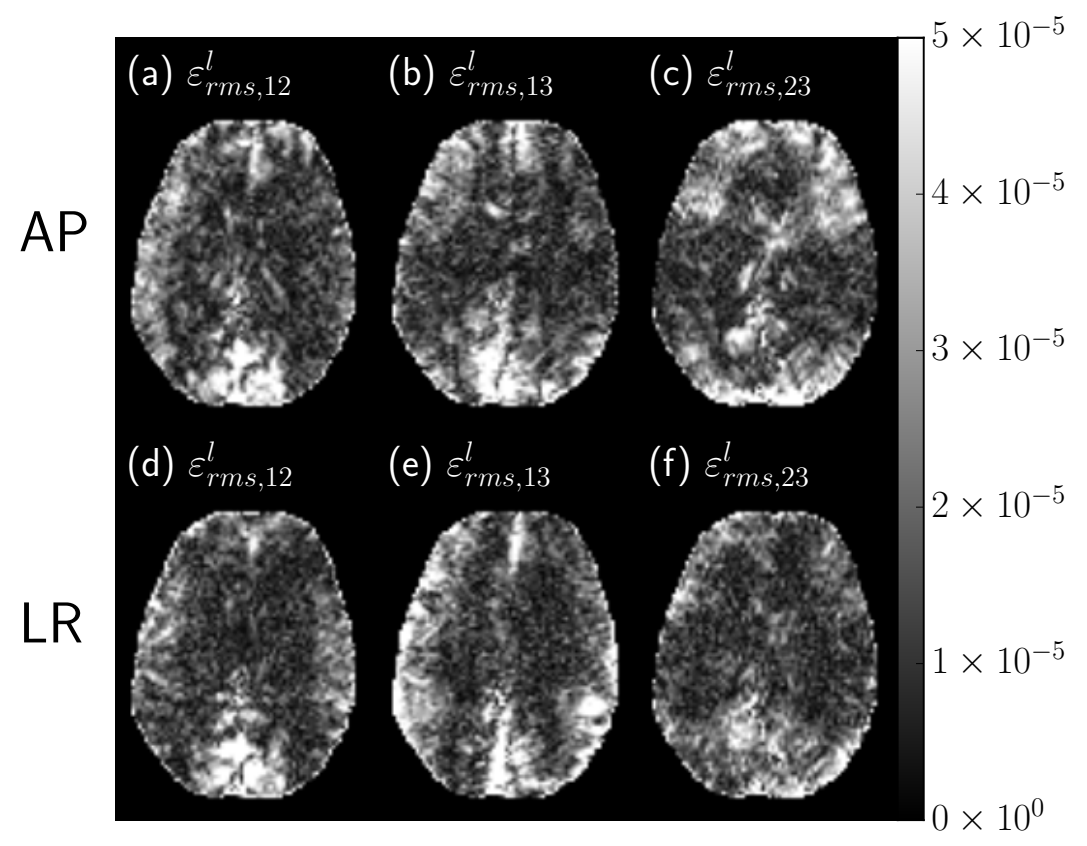

Figure 2: The RMS shear strain components of the strain tensor from both the anteriorposterior (AP) and left-right (LR) excitations. There are similar high magnitude regions for each direction but, also, distinct differences where the excitation was applied to the subject's head.

Table 2: Summary of mean and standard deviation for the reconstructed shear modulus, $G^{\prime \prime}$, across experimental repeats of spatial averages of the whole brain, global WM, and selected WM regions (CC, CR, and SLF); ()* represents $p<0.05$ for the normalized differences; $\overline{(,)}$ is a two value average.

\begin{tabular}{cccccc} 
& Whole Brain & WM & CC & CR & SLF \\
\hline $\bar{G}_{A P}^{\prime \prime}$ & $1.07(2.0 \%)$ & $1.22(2.3 \%)$ & $1.04(4.6 \%)$ & $1.49(3.1 \%)$ & $1.61(5.3 \%)$ \\
\hline $\bar{G}_{L R}^{\prime \prime}$ & $1.08(1.8 \%)$ & $1.25(2.8 \%)$ & $1.32(6.9 \%)$ & $1.84(7.1 \%)$ & $1.56(5.1 \%)$ \\
\hline$\frac{\left(\bar{G}_{A P}^{\prime \prime}-\bar{G}_{L R}^{\prime \prime}\right)}{\left(G_{A P}^{\prime \prime}, G_{L R}^{\prime \prime}\right)}$ & $-0.91 \%$ & $-2.9 \%$ & $-26 \%^{*}$ & $-33 \% *$ & $4.3 \%$
\end{tabular}



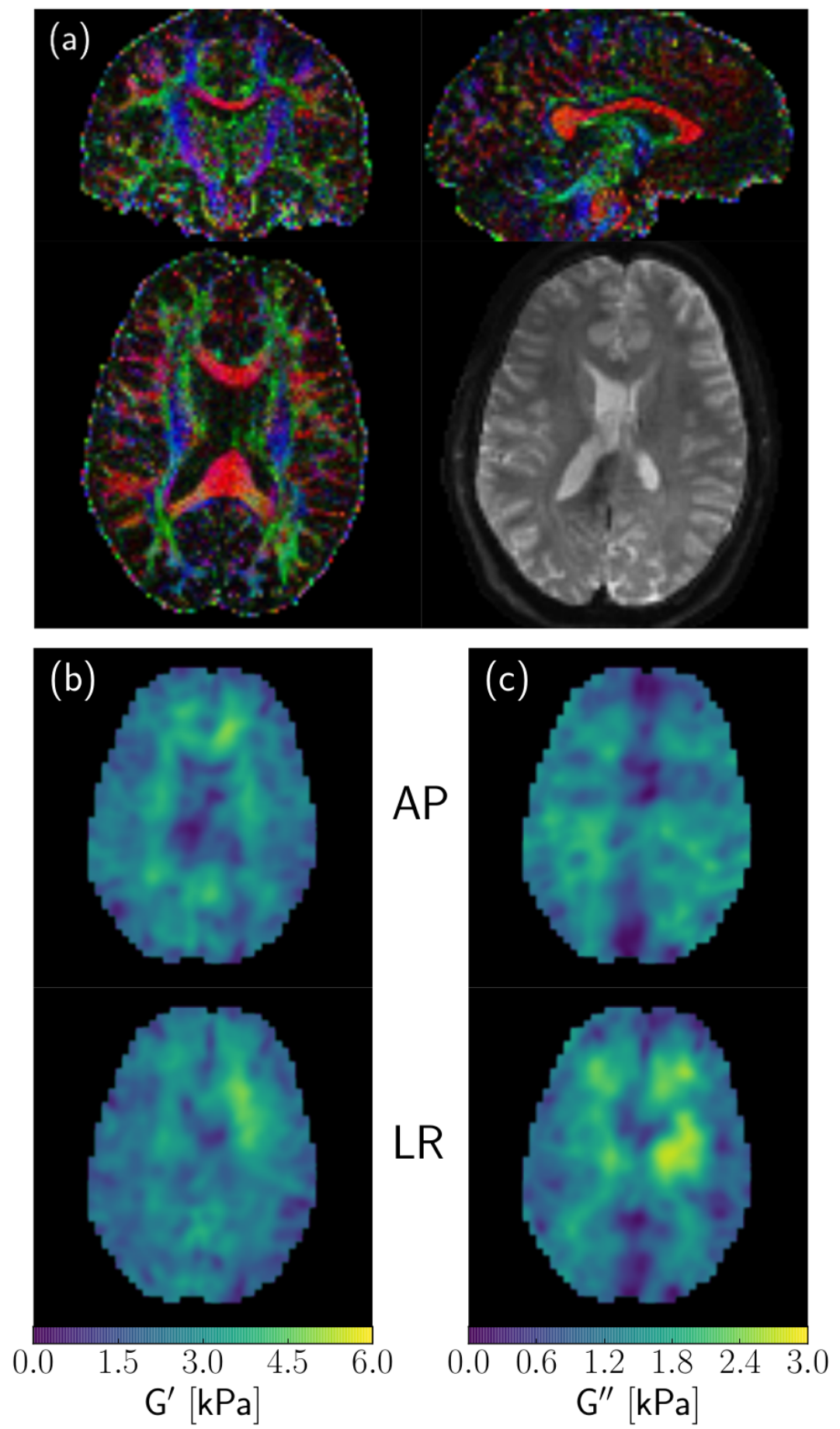

Figure 3: (a) Fractional anisotropy (FA, color intensity) from DTI (from top-left) coronal, axial and sagittal, where colors describe the fiber-tract's dominant direction: red indicates left-right, blue indicates inferior-superior, and 24 teen indicates anterior-posterior; bottom-right is a sagittal magnitude image from MRE data. The reconstructed (b) $G^{\prime}$ and (c) $G^{\prime \prime}$ material maps for anterior-posterior (AP, top) and left-right (LR, bottom) excitations. Significant differences in reconstructed values are easily identified. 


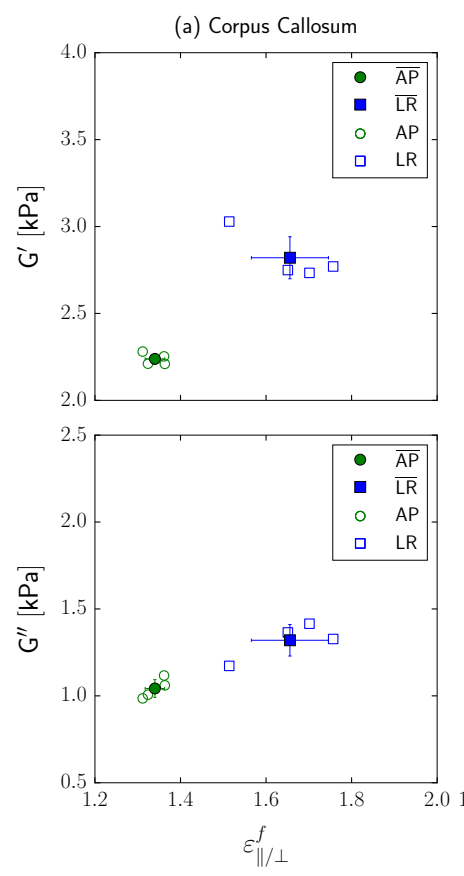

(b) Corona Radiata
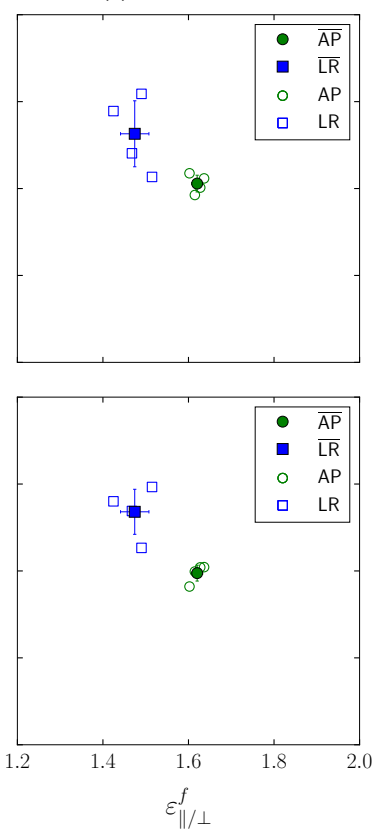

(c) Superior Longitudinal Fasciculus
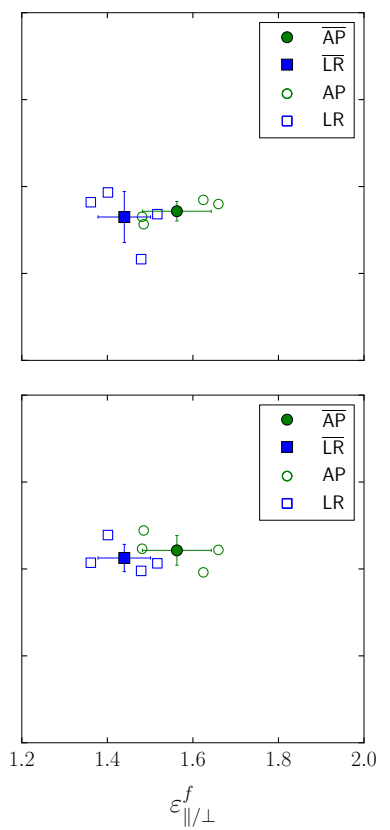

Figure 4: Comparison of reconstructed isotropic material properties, $G^{\prime}$ and $G^{\prime \prime}$, with the strain ratio of shear parallel to shear perpendicular to fibers in the fiber-tract reference frame $\varepsilon_{\| / \perp}^{f}$ (Eq. (9)) for (a) corpus callosum, CC, (b) corona radiata, CR, and (c) superior longitudinal fasciculus, SLF. The numerical values of mean and standard deviations across experimental repeats are presented in Tables 1,2 , and 3 .

Table 3: Summary of mean and standard deviation for the computed fiber-tract shear strain ratio, $\bar{\varepsilon}_{\| / \perp}^{f}$, across experimental repeats of spatial averages of the whole brain, global WM, and selected WM regions (CC, CR, and SLF); ()* represents $p<0.05$ for the normalized differences; $\overline{(,)}$ is a two value average.

\begin{tabular}{cccccc} 
& Whole Brain & WM & CC & CR & SLF \\
\hline $\bar{\varepsilon}_{\| / \perp, A P}^{f}$ & $1.61(0.23 \%)$ & $1.62(0.23 \%)$ & $1.34(2.3 \%)$ & $1.62(1.3 \%)$ & $1.56(8.0 \%)$ \\
\hline $\bar{\varepsilon}_{\| / \perp, L R}^{f}$ & $1.59(0.54 \%)$ & $1.56(0.30 \%)$ & $1.66(9.0 \%)$ & $1.47(3.3 \%)$ & $1.44(6.1 \%)$ \\
\hline$\frac{\left(\bar{\varepsilon}_{\| / \perp, A P}^{f}-\bar{\varepsilon}_{\| / \perp, L R}^{f}\right)}{\left(\varepsilon_{\| / \perp, A P}^{f}, \varepsilon_{\| / \perp, L R}^{f}\right)}$ & $1.3 \%$ & $3.4 \%$ & $-22.3 \% *$ & $8.8 \% *$ & $7.5 \%$
\end{tabular}

